\title{
Network-based approaches for anticancer therapy (Review)
}

\author{
HYUNJEONG SEO, WANYEON KIM, JIHYUNG LEE and BUHYUN YOUN \\ Department of Biological Sciences, College of Natural Sciences, Pusan National University, \\ Busan 609-735, Republic of Korea
}

Received June 27, 2013; Accepted August 23, 2013

DOI: 10.3892/ijo.2013.2114

\begin{abstract}
Cancer is a complex disease resulting from alterations of multiple signaling networks. Cancer networks have been identified as scale-free networks and may contain a functionally important key player called a hub that is linked to a large number of interactors. Since a hub can serve as a biological marker in a given network, targeting the hub could be an effective strategy for enhancing the efficacy of cancer treatment. Chemotherapies and radiotherapies are generally used to treat tumors not amenable to resection, and target single or multiple molecules associated with hubs. However, these therapies may unexpectedly induce the resistance of cancer cells to drugs and radiation. Cancer cells can overcome therapy-induced damage via the activation of back-up signaling pathways and flexible modulation of affected networks. These activities are considered to be the main reasons for chemoresistance and radioresistance, and subsequent failure of cancer therapies. Much effort is required to identify the key molecules that control the modulation of signaling networks in response to drugs and radiation. Network-based therapy that affects network flexibility, including rewired network structures and hub molecules in these networks, could minimize the occurrence of side-effects and be a promising strategy for enhancing the therapeutic efficacy of cancer treatments. This review is intended to offer an overview of current research efforts including ones focused on cancer-associated complex networks, their modulation in response to cancer therapy, and further strategies targeting networks that may improve cancer treatment efficacy.
\end{abstract}

\section{Contents}

1. Introduction

2. Complex networks in biology

3. Cancer-associated networks

Correspondence to: Dr BuHyun Youn, Department of Biological Sciences, College of Natural Sciences, Pusan National University, Busandaehak-ro 63, Geumjeong-gu, Busan 609-735, Republic of Korea E-mail: bhyoun72@pusan.ac.kr

Key words: complex network, hub, network flexibility, chemotherapy, radiotherapy
4. Network-based approaches for chemotherapy

5. Network-based approaches for radiotherapy

6. Targeting network flexibility

7. Conclusion

\section{Introduction}

Cancer is characterized as a complex and heterogeneous disease involving an orchestration of distinct cellular signaling events that can be affected by the aberrant expression or mutation of genes and chromosomes, tumor microenvironments and tissue origin of the tumor. Experimental and literature-based analyses of signaling networks supported the hypothesis that a common network and its components are the same for all cells (1-3). Therefore, it has been proposed that differentially expressed tumor phenotypes might result from distinct interactions and consequent activation of specific subnetworks (2). Thus, single-target therapies using highly specific compounds will likely fail as a cancer treatment unless the compounds are able to disrupt an actual network. In order to deal with this problem, network-based approaches have emerged (4). Most cellular components interact with each other to carry out biological functions within the same cell or between cells. These intra- and intercellular interactions form a complex and flexible network, and are dynamically altered on temporal and spatial scales. This is responsible for the determination of tumor phenotypes. Therefore, cancer-associated molecular networks and their dynamics could be potential targets for therapeutic intervention. With chemo- and radiotherapies, dynamic alteration of signaling networks occurs in tumor cells as protective processes against stress stimuli. These cellular responses are the main cause of resistance to therapies. To develop better cancer treatment strategies, it is important to determine which subnetworks are activated and which factors play a crucial role in network alteration upon chemo- and radiotherapies. In this review, we will describe the complex networks associated with cancers, their properties, and further strategies targeting these networks for development of efficient anticancer therapies.

\section{Complex networks in biology}

Cells can be depicted as complex networks of macromolecular interactions (5). Most biological processes are executed through multi-scale dynamic complex systems formed by interacting macromolecules, metabolites, cells and tissues (6). 
At a highly abstract level, components of a network can be reduced to a series of nodes that are interconnected by several links with each link representing the interactions between two components (7). Nodes are basic components of a network and are connected by links. In biological networks, proteins, metabolites, DNA and RNA correspond to nodes and their interactions represent links.

Several model organisms have produced a bulk of information for understanding biological networks (8). Based on these data, several studies examining human-specific networks have been recently performed. Many groups have focused on molecular networks such as protein-protein interaction networks, metabolic networks, DNA/RNA networks and gene regulatory networks. In networks of protein-protein interaction, proteins serve as nodes and are linked to each other by physical interactions (8-10). Metabolic networks consist of metabolites as nodes that are linked if they play a role in the same biochemical pathway $(11,12)$. In DNA/RNA networks, regulatory RNA molecules (such as microRNA and small interfering RNA) and DNA are considered nodes and their links indicate functional interactions that influence the regulation of gene expression (13-15). In gene regulatory networks, direct links of each node represent the regulatory relationships between a transcriptional factor and a gene $(13,16)$. Each of these networks is fundamentally present in all cells (3). However, these networks are differentially modulated in a cell type-specific manner. Due to the complexity and dynamics of the networks, cells are able to develop unique phenotypes (2). It means that each cell may distinctively respond to the same stimulus. Thus, it is encouraged to understand the common network structures and their signaling process by downstream effectors that are associated with specific phenotype, which can be applied to the implication of cancer cell-specific responses to targeted therapies.

Network structures are divided into two major classes based on the distribution of connections, indicating the probability that one node in the network is linked to other nodes. One class of networks is characterized as a random network, and follows a Poisson distribution (Fig. 1A). In a random network, most nodes generally have the same number of links, resulting in a fairly homogeneous network. In contrast, many real systems including biological networks belong to a class of heterogeneous networks called scale-free networks (Fig. 1B). This type of network follows a power-law for which a few nodes have a lot of links and most nodes have a few links (1 or 2 links) (17,18). The few highly connected nodes are known as hubs. Perturbing these hubs could be catastrophic since they render the whole network structure more stable and robust (19). Ironically, existence of hubs could cause network fragility by offering reasonable targets for attacks. However, natural events that cause network disruption (such as mutations) are classified as failures rather than attacks since these are non-specific events. In the absence of specific attacks, hubs are relatively free from damage owing to dilution effect of a number of non-hub nodes when a given network is disrupted in a non-specific or random manner. Due to the existence of hubs, networks can display a robustness to random errors (20).

As mentioned above, most biological networks characterized as heterogeneous scale-free networks have the unique property of possessing hubs. In a scale-free network, hub disruption can lead to a major loss of connectivity that induces network disturbance (21). Due to this property, it is very likely that the proteins identified as hubs are critical players in various biological processes. In particular, studies using model organisms have shown that hub proteins are more closely associated with essential genes. Due to their functions and essentiality, the genes encoding hub proteins also tend to be more conserved and evolve more slowly than ones encoding non-hub proteins $(19,22)$. In addition to protein networks, disruption of the networks containing essential metabolites (hub metabolites) could cause severe cellular damage that would negatively impact cell survival and growth. However, when the processes involving nonessential metabolites are interfered with, cellular functions are not significantly affected (23). These data demonstrate that targeting hubs can cause considerable damage to an overall cellular network compared to targeting non-hub molecules. Another study was conducted to identify cancer-specific network signatures using proteomic analysis (24). Several proteins including PTEN, cyclin B1, p-CREB and vascular endothelial growth factor (VEGF) were considered to be hubs of cancer-associated networks, which could provide the implication to cancer diagnostic markers, prognostic markers and potential therapeutic targets. Thus, extracting hub elements could serve as an invaluable strategy for drug discovery. The importance of hubs is also emphasized by their potential use as biological markers.

Based on spatial and chemical connections for cellular functionality in a process, a biological network is subdivided in a modular manner $(25,26)$. Several functional modules (subnetworks) could determine phenotypic changes. In disease processes, these modules are called disease modules, and their development is associated with disease occurrence (27). Since various modules composed of a complex web of node interactions are densely integrated, modular organization for a disease process is not immediately apparent in a whole network and it is difficult to specify a disease-associated module correctly. Previous studies have been conducted to characterize specific networks and their hubs, including ones associated with cancer, that might be used as disease markers (28-32). Furthermore, disease incidence, state and severity could be evaluated based on network signatures. Altogether, identification of the hub molecules in biological complex networks is important because these hubs could serve as specific biomarkers and drug targets.

\section{Cancer-associated networks}

Cancer is one of complex diseases caused by alterations of various signaling networks responsible for major cellular functions such as proliferation, survival and apoptosis. These complex networks consisting of several signaling modules are driven by dynamics of various components, including DNA, RNA, and proteins, as well as their connections leading to many intracellular pathways such as crosstalk and feedback loops in response to internal and external stimuli. Components in each module function within different temporal and spatial scales, leading to cancer heterogeneity. The integration of dynamic signaling modules ultimately influences cell phenotype and 


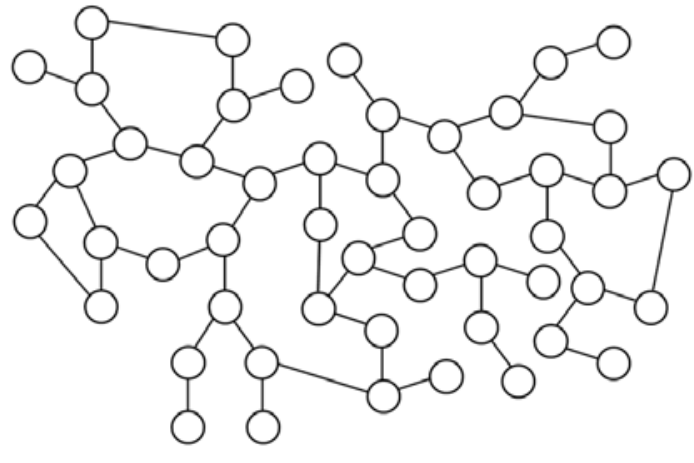

(A) Random network

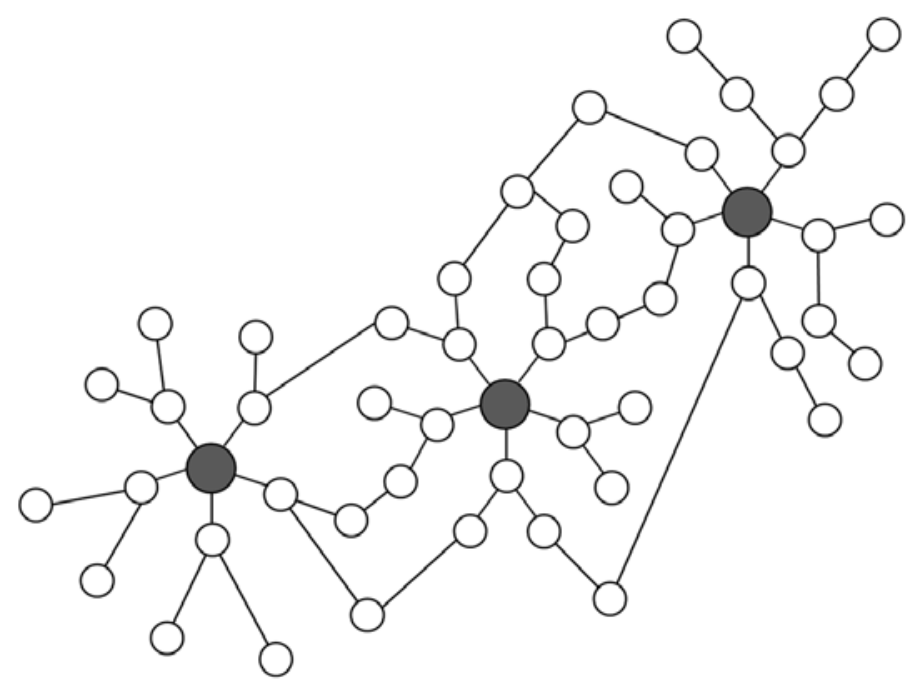

(B) Scale-free network

Figure 1. The random network and scale-free network. (A) Random network follows Poisson distribution and is nearly homogenous. Most nodes have approximately the same numbers of links. The road system, for example, fits with this type of network model. (B) Scale-free network follows power-law and is inhomogeneous. Most nodes have one or two links but a few highly-connected nodes, so-called hubs, have a large number of links. The airline network and most of spontaneously-generated networks, for example, follow scale-free network. White circles indicate nodes and dark ones indicate hubs that are highlyconnected nodes.

may result in tumorigenesis. Thus, understanding the complex networks and their modules associated with cancer can be promising for the development of novel therapeutic strategies.

Much effort has been devoted to understanding tumorassociated networks involved in tumor initiation, progression, and metastasis using various high-throughput techniques (microarray, next-generation sequencing, and 2-dimensional electrophoresis/mass spectrometry) and bioinformatics (computational algorithms and statistical/analytical tools). Detailed interactome maps of several tumor types may help identify network nodes as potential targets for therapeutic strategies that are more effective than traditional approaches such as gene-focused therapies which hardly consider the biological context of the targets (31,33-36). Out of approximately 25,000 human genes, only hundreds are considered to represent essential diseases genes. A large proportion of these genes has been identified as tumor-associated hubs and includes ones encoding epidermal growth factor receptor (EGFR), Ras, Akt, PTEN and p53 (37-40). EGFR network is important for tumor growth, progression and metastasis in human cancers, since EGFR have been identified to play a critical role in DNA repair, cell cycle progression, proliferation, and cell motility (39). It has been reported that EGFR mutations were accumulated in patients with non-small cell lung cancer and these mutant EGFR could be indicators for tumor behavior and poor prognosis (41). In case of p53, a tumor suppressor gene, DNA damage response can induce the expression of this gene, consequently leading to cell cycle arrest and apoptosis. p53 has been identified to have loss-of-function mutations in various types of cancers (37). Furthermore, it is very likely that disruption of p53 is highly associated with tumor initiation and development (42). These hub genes usually have been revealed to be dysregulated in many cancers, leading to hyper-activation of proliferative networks, distant metastatsis, and evasion of apoptotic cell death. Due to the functional importance of hubs in cellular systems, modulation of such hub networks is highly responsible for decision of cancer phenotypes. In addition, these hubs may serve to the clinical implications in promising design for therapeutic strategy.

\section{Network-based approaches for chemotherapy}

Over the past decades, chemotherapeutic drug discovery and development have focused on specific inhibitors that target hub or hub-associated proteins (Table I). Although these strategies initially increased curative efficacy with great potency of targeting hub element in a given disease network, these target therapies have generally been inappropriate for cancer treatment due to their side-effects such as the induction of drug resistance. It is very likely that network structures have remarkable flexibility achieved through the alteration of subnetworks, including pathway reprogramming and activation of a crosstalk pathway, in response to external stimuli $(43,44)$. Indeed, biological components are highly redundant due to gene duplication and the existence of protein isomers and families, which have different properties despite the inclusion of very closely related proteins. This redundancy enables the maintenance of an entire biological network through the activation of compensatory or detouring networks in response to stress-induced damage (45-47). For example, the MEK kinase inhibitor GSK1120212 inhibits MEK1 but not MEK2 that lacks a binding site for the inhibitor (48). Therefore, MEK2 could escape drug-induced inhibition. Undisturbed MEK2 could then reactivate the pathway blocked by GSK1120212 via the activation of mediator proteins instead of MEK1. This event is a consequence of network reprogramming (49). In another study, herceptin was devel- 
Table I. Hub elements and their functions in cancer-related networks.

\begin{tabular}{|c|c|c|c|}
\hline Hub element & Biological effect & Targeted drug & Refs. \\
\hline VEGF & Invasion, angiogenesis, metastasis & Bevacizumab, Ranibizumab & $(24,54,71-73)$ \\
\hline EGFR/Her2 & $\begin{array}{l}\text { Proliferation, invasion, metastasis, } \\
\text { cell cycle progression, DNA repair, } \\
\text { anti-apoptosis }\end{array}$ & $\begin{array}{l}\text { Lapatinib, Erlotinib, Cetuximab, } \\
\text { Trastuzumab }\end{array}$ & $(39,41,50-53,77,78)$ \\
\hline $\mathrm{NF}-\kappa \mathrm{B}$ & $\begin{array}{l}\text { Inflammation, proliferation, survival, } \\
\text { radioresistance }\end{array}$ & Denosumab (RANKL inhibitor) & (70) \\
\hline PI3K/Akt & $\begin{array}{l}\text { Proliferation, metabolism, survival, } \\
\text { anti-apoptosis }\end{array}$ & $\begin{array}{l}\text { GS-1101 (phase II), PX-866 (phase II), } \\
\text { KRX-0401 (phase III) }\end{array}$ & $(53,54)$ \\
\hline HIF-1 & $\begin{array}{l}\text { Hypoxia response, glycolytic switch, } \\
\text { survival, invasion, angiogenesis, } \\
\text { metastasis }\end{array}$ & $\begin{array}{l}\text { EZN-2208 (phase I), EZN-2968 (phase I), } \\
\text { PX-478 (phase I) }\end{array}$ & $(54,71-73)$ \\
\hline p53 & $\begin{array}{l}\text { Tumor suppressor activity, DNA repair, } \\
\text { cell cycle arrest, senescence, apoptosis }\end{array}$ & & $(37,42)$ \\
\hline PTEN & $\begin{array}{l}\text { Senescence, anti-proliferation, } \\
\text { tumor suppressor activity }\end{array}$ & & (24) \\
\hline
\end{tabular}

oped for targeting cases of breast cancer that express HER2, a member of the EGFR family $(50,51)$. Herceptin-resistance is sometimes acquired during treatment due to the activation of other signaling subnetworks, including the Akt-induced glycolytic pathway or Bcl-2-mediated anti-apoptotic pathway, as compensatory crosstalk networks $(52,53)$. Additionally, for treating metastatic renal cell carcinomas, therapies that target both the VEGF receptor (VEGFR) and the mammalian target of rapamycin complex 1 (mTORC1) showed better therapeutic efficiency than previous traditional treatments (54). Unexpectedly, cancer cells have acquired resistance to the combination therapies via inhibition of VEGFR and mTORC1 and patients eventually suffered from tumor relapse with re-established tumor vasculature. Acquiring drug-resistant mechanisms was accomplished by the loss of negative feedback networks involving suppression of mTORC2 and Akt signaling, which could consequently result in mTORC2mediated signaling activation as a crosstalk network for drug-induced mTORC1 inhibition. This event caused the mTORC2-mediated Akt and hypoxia-inducible factor-1 (HIF-1) activation for angiogenesis, leading to poor prognosis in metastatic renal cell carcinomas.

These findings demonstrate that altered network states including network reprogramming and the existence of crosstalk network can complement a hub-associated network disrupted by a specific drug, and are major causes of drug resistance. Single-target drugs might not only have beneficial effects on dysfunctional aspects of disease-associated modules in entire complex networks of cancer, but they could also turn-on other components in nearby disease-associated modules showing side-effects.

\section{Network-based approaches for radiotherapy}

Radiotherapy is one of the major modalities of cancer management. More than $50 \%$ of the patients with cancer have undergone radiation treatment. Ionizing radiation (IR) gener- ates intermediate free radicals and reactive oxygen species leading to DNA double-strand breaks (DSBs) (55). Unless cells repair this type of injury properly, they directly or indirectly undergo cell death. Although many tumor cells immediately die via apoptosis after radiation exposure, some of cells can survive through activation of DSB repair pathway including homologous recombination and non-homologous end-joining $(56,57)$. The surviving tumor cells accompany unavoidable gene mutations due to the properties of DSB repair modules, leading to additional effects including hyper-activation of crosstalk signaling to compensate the loss of a certain gene that account for radioresistance. Indeed, other modules are also activated and integrated for helping tumor cells overcome IR-induced stress. It is supported by several interactome and gene profiling analyses using various types of cancer cells treated with radiation (58-63). The results indicated that numerous cellular networks, including modules for DNA repair, survival, apoptosis, cell cycle, cell migration, protein localization, RNA processing, antioxidant defense, inflammation and cell proliferation, are altered by radiation exposure and help determine tumor cell fate. For example, p53-related genes and DNA-damage response genes are generally activated by irradiation in susceptible lung cancer cells while the networks associated with these genes are disrupted in radioresistant lung cancer cells $(60,62,63)$.

In addition to drug-induced network alterations, radiotherapy could also contribute to the activation of new subnetworks, resulting in network flexibility (64-66). Unlike drugs which act on specific target molecules, ionizing radiation exerts effects on whole cell components (67). Thus, the proportion of hub elements that are functionally disrupted by radiotherapy is relatively low. This is due to the dilution effect of non-hub elements since these elements are more abundant and consequently subjected to greater damage (18). Hubs that remain undamaged could eventually activate other radiationresponsive signaling networks, reintegrate network topologies and establish networks more resistant to radiotherapy, thus 
leading to radioresistance $(68,69)$. For example, NF-кBmediated inflammatory signaling cascade in cancer cells could be activated in response to reactive oxygen species generated by irradiation (70). Activated inflammatory network with various chemokines and cytokines is associated with cancer cell survival activity for overcoming radiotherapy-induced inflammatory stress. This event might result in acquired radioresistance in cancer cells and consequently reduced efficacy of following radiotherapy in tumor. During tumor development, cancer cells partially undergo hypoxic condition due to insufficient vasculature systems. Thus, these cells show hyperactivation of HIF-1 network for adaptation to hypoxic stress. It has been well-studied that cancer cells show more resistance to irradiation under hypoxia than under normoxia (71). Several studies have revealed that radiation induced HIF-1 stabilization and activate its signaling module in a solid tumor via oxidative stress leading to the increase in VEGF expression, which is well-known to play a protective role in endothelial cells from the radiation-induced cytotoxic effects $(72,73)$. Consequently, tumor cells can be supplied oxygen and nutrients from the protected tumor vasculature, leading to tumor radioresistance and tumor growth progression.

Since exposure of tumor cells to radiation could impact a large number of proteins simultaneously, it might take much time and effort to identify hubs as specific drug targets among the proteins affected by IR. Instead, understanding the altered activation patterns of various IR-responsive modules could allow us to hypothesize which proteins are responsible for critical functions in each module. This will help identify hubs and consequently promote the development of novel radiotherapy strategies. Moreover, therapeutic efficacy could be improved by administrating radiotherapy in conjunction with chemotherapeutic agents such as radiosensitizers or inhibitors targeting hub molecules associated with radioresistance.

\section{Targeting network flexibility}

As mentioned above, a large number of traditional strategies that do not account for the dynamics of complex systematic networks are not satisfactory cancer treatments. Biological networks in tumors gradually adapt to chemo- and radiotherapy. Tumor cells are thus able to maintain their tumorigenic properties through compensatory mechanisms such as crosstalk circuits $(74,75)$. To improve cancer therapeutic efficacy, targeting the hub itself might be insufficient. Additionally, hub-associated network flexibility developed in response to therapeutic challenges could be targeted. Consequently, the field of network medicine has recently emerged $(4,27,43)$. Based on numerous system biological studies, several investigations have been recently conducted to identify and discover ways to regulate back-up networks activated during chemotherapy, and develop network medicine to overcome chemoresistance $(28,76,77)$. For the patients with Her2-positive breast cancer, lapatinib was approved as the first dual inhibitor of EGFR/Her2. However, the efficiency of this drug was not prolonged due to acquired resistance. A network-based computational analysis showed that, while lapatinib initially induced inhibition of glucose uptake and energetic stress leading to apoptosis in Her2-positive cancer cells, the glucose deprivation response network is gradually activated as a compensatory mechanism in response to the inhibition of the Her2-mediated oncogenic network by lapatinib, which eventually result in drug resistance (Fig. 2) (77). It was suggested that novel combinations should be administered to simultaneously target Her2 networks and metabolic networks to treat cases of Her2-positive breast cancer that had acquired drug resistance. For the patients with hormone receptor-positive metastatic breast cancer, letrozole, an inhibitor for aromatase (estrogen producing enzyme), could be treated for endocrine therapies (78). However, this endocrine therapy showed unsatisfactory effect due to the activation of cross-talk pathways involving EGFR/Her2 and estrogen receptor (ER), leading to resistance to therapies. It was presented that the combined therapies with lapatinib (or herceptin) and letrozole could be a promising strategy for endocrine resistance by blocking ER-mediated hormone signaling as well as compensatory EGFR/Her2 networks for survival signaling.

When it comes to radiotherapy, complex networks that are critically responsible for network flexibility resulting in acquired radioresistance are not fully understood. Nevertheless, some investigations have demonstrated the involvement of several genes, including HDAC1, MDM2, c-Jun, PKC- $\beta, c-A b l$ and CDK1, in cellular responses to radiation (79-81). Some of these genes have been studied as potential targets for radiosensitizer development. For example, c-Abl, a non-receptor tyrosine kinase, plays a critical role in cell survival, proliferation, and anti-apoptotic activity, leading to tumorigenesis. In addition to oncogenic properties of c-Abl, a study using glioma cells showed that c-Abl elevated the expression of Rad51 in response to radiation, which is a crucial component of the DNA repair pathway, especially DSBs (82-84). It means that c-Abl could modulate radio-response through activating DNA repair module leading to radioresistance. In this case, STI571, a pharmacological drug of c-Abl kinase, could be used to block c-Abl-Rad51 signaling for a DNA repair module to render radiosensitizing effect in glioma cells, but this drug had no effect in normal cells (82). It could be concluded that several compensatory modules such as the cell cycle module, proliferative module, and DNA repair module are activated after radiotherapy to protect tumor cells against IR-induced injuries. Activation of these modules would lead to acquired radioresistance, cancer cell survival, and tumor re-growth. Thus, it is necessary to use network-specific drugs as radiotherapeutic adjuvants that suppress the activity of survival-associated modules and prevent unexpected side-effects. In order to prevent tumor cells from acquiring resistance to chemo- and radiotherapy, it is important to understand the rewiring states of networks and their essential nodes in response to cancer therapies. In this manner, a network-based combination therapy targeting the hubs associated with network flexibility can be formulated to overcome adverse effects induced by current therapies.

\section{Conclusion}

Network-based therapies for treating human cancers may have various promising biological and clinical applications. In particular, hub elements in a disease network could function as biological markers because these hubs are highly connected to biological scale-free networks and their roles in each network are 
(A)

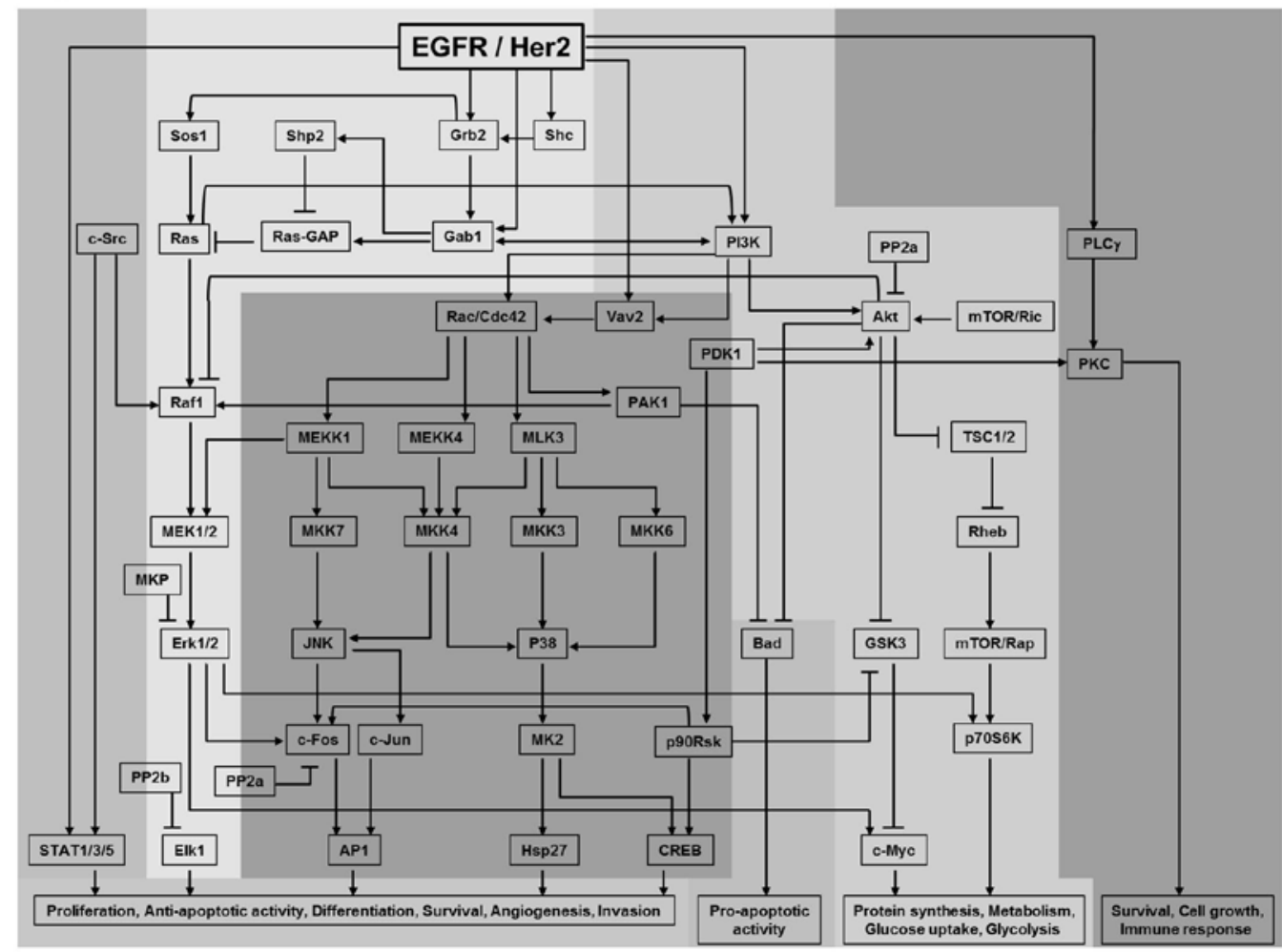

(B)

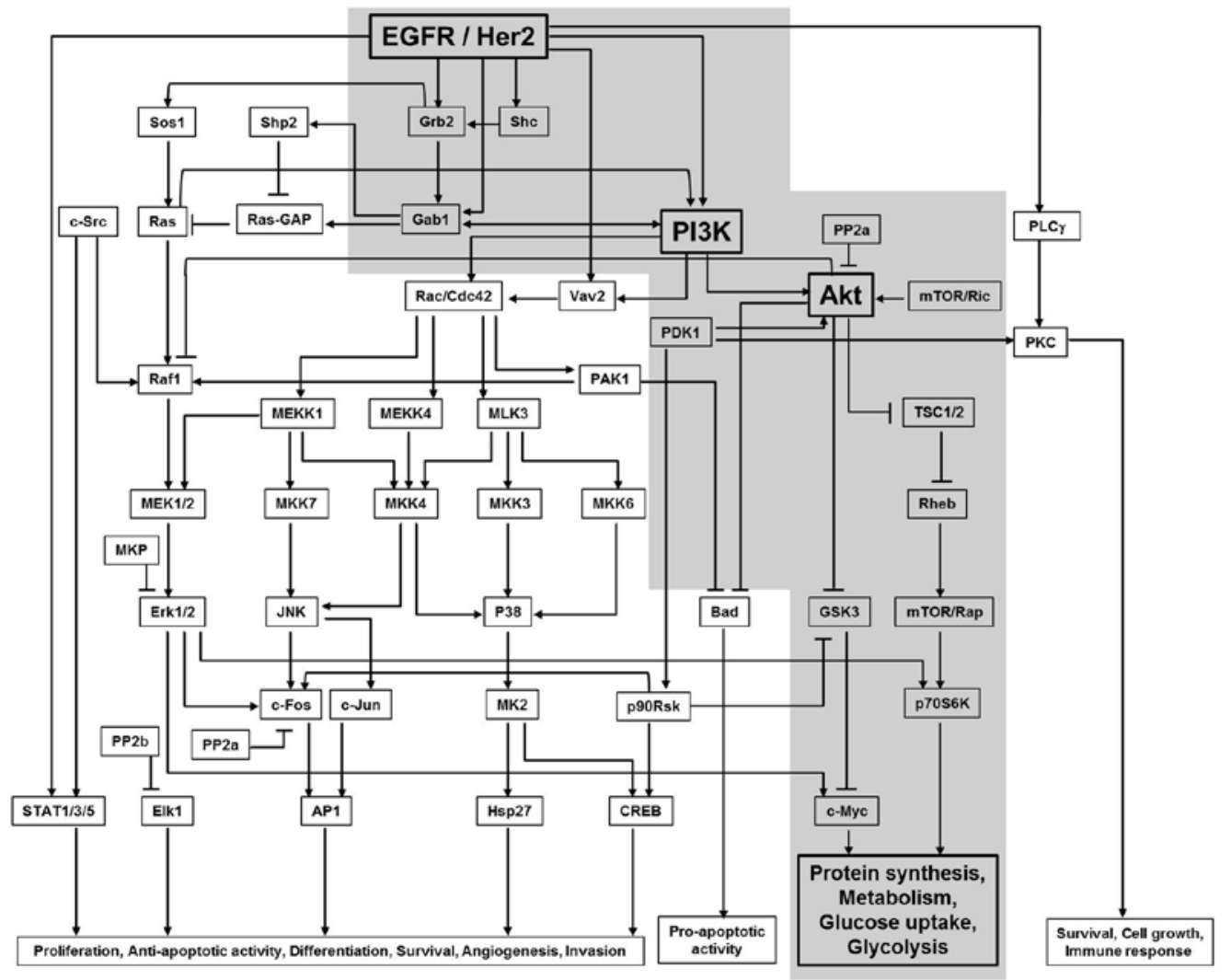

Figure 2. An EGFR network structure. (A) An EGFR network including several functional modules in cells is presented. EGFR and Her2 as well-known receptor tyrosine kinases can activate various downstream effectors in response to external stimuli, including their ligands, drugs and radiation. Consequently, many functional signaling modules are influenced, which are usually associated with tumor initiation and development, including cell survival, metabolism activation, cell cycle progression, proliferation and differentiation. EGFR/Her2-targeted drugs might interrupt some of these modules, leading to tumor cell death and therapeutic effects. (B) A compensatory module in response to lapatinib in drug-resistance cancer cells is presented as a shaded area. Lapatinib is one of EGFR/Her2 inhibitors. Although the tumor shrinkage efficiency was shown in Her2-positive breast cancer patients, it could not be repeatedly administered in cancer therapy due to acquired drug-resistance. The reason is that lapatinib-induced glucose deprivation, which led to tumor cytotoxicity, might activate a cross-talk module for the increase of glucose uptake and metabolism to adapt to stress condition, leading to drug-resistance and poor tumor prognosis. 
essential. It is probable that the fate of tumor cells is controlled by the regulation of hub elements. Analysis of biological networks and the discovery of hub elements will help identify novel drug-targets as well as diagnostic markers for detecting early stage cancer. Single-target therapies might take advantage of some aspects of the disease modules associated with cancer, but these modalities are not generally effective since complex biological networks consisting of various disease modules exist in tumor cells and tissues. In addition, simple multi-target therapies are still not optimal cancer treatments because biological networks are dynamically altered in a stimulus-dependent manner to maintain homeostasis (in this case, cancer cell homeostasis for survival and proliferation). Biological systems are highly heterogeneous and network structures in the context of tumors are flexible enough for adaptation to various external stimuli. Network-based combinational approaches could be the most promising strategies for silencing specific mediators (i.e., novel hubs) responsible for the alteration of network states. These techniques could maximize the effect of more traditional therapies by simultaneous administration of pharmacological agents that specifically target hubs and disrupt major disease modules. A large quantity of integrated bioinformatics data has been gradually collected over time. Using this information, we can closely examine entire network structures and their states. In addition, it will be possible to predict how a network state will be modified in response to chemo- and radiotherapy. This will facilitate the development of ideal network-based drug combinations and personalized therapeutic strategies.

\section{Acknowledgements}

This study was supported by the Bio-Scientific Research Grant funded by the Pusan National University (PNU-2010-101-249).

\section{References}

1. Janes KA and Lauffenburger DA: A biological approach to computational models of proteomic networks. Curr Opin Chem Biol 10: 73-80, 2006.

2. Miller-Jensen K, Janes KA, Brugge JS and Lauffenburger DA: Common effector processing mediates cell-specific responses to stimuli. Nature 448: 604-608, 2007.

3. Jordan JD, Landau EM and Iyengar R: Signaling networks: the origins of cellular multitasking. Cell 103: 193-200, 2000.

4. Pawson T and Linding R: Network medicine. FEBS Lett 582: 1266-1270, 2008.

5. Vidal M, Cusick ME and Barabasi AL: Interactome networks and human disease. Cell 144: 986-998, 2011.

6. Vidal M: A unifying view of 21 st century systems biology. FEBS Lett 583: 3891-3894, 2009.

7. Barabasi AL and Oltvai ZN: Network biology: understanding the cell's functional organization. Nature Rev Genet 5: 101-113, 2004.

8. Ideker T and Sharan R: Protein networks in disease. Genome Res 18: 644-652, 2008.

9. Rual JF, Venkatesan K, Hao T, et al: Towards a proteome-scale map of the human protein-protein interaction network. Nature 437: 1173-1178, 2005.

10. Stelzl U, Worm U, Lalowski M, et al: A human protein-protein interaction network: a resource for annotating the proteome. Cell 122: 957-968, 2005.

11. Jeong H, Tombor B, Albert R, Oltvai ZN and Barabasi AL: The large-scale organization of metabolic networks. Nature 407: 651-654, 2000

12. Chang RL, Xie L, Bourne PE and Palsson BO: Drug off-target effects predicted using structural analysis in the context of a metabolic network model. PLoS Comput Biol 6: e1000938, 2010.

13. Schlitt T and Brazma A: Current approaches to gene regulatory network modelling. BMC Bioinformatics 8 (Suppl 6): S9, 2007.
14. Zhao Y, He S, Liu C, et al: MicroRNA regulation of messengerlike noncoding RNAs: a network of mutual microRNA control. Trends Genet 24: 323-327, 2008.

15. He X, He L and Hannon GJ: The guardian's little helper: microRNAs in the p53 tumor suppressor network. Cancer Res 67: 11099-11101, 2007.

16. Zhou Q, Chipperfield H, Melton DA and Wong WH: A gene regulatory network in mouse embryonic stem cells. Proc Natl Acad Sci USA 104: 16438-16443, 2007.

17. Barabasi AL and Albert R: Emergence of scaling in random networks. Science 286: 509-512, 1999.

18. Albert R, Jeong $\mathrm{H}$ and Barabasi AL: Error and attack tolerance of complex networks. Nature 406: 378-382, 2000.

19. Jeong H, Mason SP, Barabasi AL and Oltvai ZN: Lethality and centrality in protein networks. Nature 411: 41-42, 2001.

20. Park J and Newman ME: Statistical mechanics of networks Phys Rev E Stat Nonlin Soft Matter Phys 70: 066117, 2004.

21. Albert R: Scale-free networks in cell biology. J Cell Sci 118: 4947-4957, 2005

22. Fraser HB, Hirsh AE, Steinmetz LM, Scharfe C and Feldman MW: Evolutionary rate in the protein interaction network. Science 296: 750-752, 2002.

23. Kim PJ, Lee DY, Kim TY, et al: Metabolite essentiality elucidates robustness of Escherichia coli metabolism. Proc Natl Acad Sci USA 104: 13638-13642, 2007.

24. Zhang DY, Ye F, Gao L, et al: Proteomics, pathway array and signaling network-based medicine in cancer. Cell Div 4: 20 , 2009.

25. Ravasz E, Somera AL, Mongru DA, Oltvai ZN and Barabasi AL: Hierarchical organization of modularity in metabolic networks. Science 297: 1551-1555, 2002.

26. Han JD, Bertin N, Hao T, et al: Evidence for dynamically organized modularity in the yeast protein-protein interaction network. Nature 430: 88-93, 2004.

27. Barabasi AL, Gulbahce N and Loscalzo J: Network medicine: a network-based approach to human disease. Nature Rev Genet 12: 56-68, 2011.

28. Creixell P, Schoof EM, Erler JT and Linding R: Navigating cancer network attractors for tumor-specific therapy. Nat Biotechnol 30: 842-848, 2012.

29. Hwang S, Son SW, Kim SC, Kim YJ, Jeong H and Lee D: A protein interaction network associated with asthma. J Theor Biol 252: 722-731, 2008.

30. Jonsson PF and Bates PA: Global topological features of cancer proteins in the human interactome. Bioinformatics 22: 2291-2297, 2006.

31. Chuang HY, Lee E, Liu YT, Lee D and Ideker T: Network-based classification of breast cancer metastasis. Mol Syst Biol 3: 140, 2007.

32. Garcia M, Millat-Carus R, Bertucci F, Finetti P, Birnbaum D and Bidaut G: Interactome-transcriptome integration for predicting distant metastasis in breast cancer. Bioinformatics 28: 672-678, 2012

33. Pache RA, Zanzoni A, Naval J, Mas JM and Aloy P: Towards a molecular characterisation of pathological pathways. FEBS Lett 582: 1259-1265, 2008.

34. Chand Y and Alam MA: Network biology approach for identifying key regulatory genes by expression based study of breast cancer. Bioinformation 8: 1132-1138, 2012.

35. Sonachalam M, Shen J, Huang H and Wu X: Systems biology approach to identify gene network signatures for colorectal cancer. Front Genet 3: 80, 2012.

36. Breitkreutz D, Hlatky L, Rietman E and Tuszynski JA: Molecular signaling network complexity is correlated with cancer patient survivability. Proc Natl Acad Sci USA 109: 9209-9212, 2012.

37. Vogelstein B, Lane D and Levine AJ: Surfing the p53 network. Nature 408: 307-310, 2000.

38. Goh KI, Cusick ME, Valle D, Childs B, Vidal $M$ and Barabasi AL: The human disease network. Proc Natl Acad Sci USA 104: 8685-8690, 2007.

39. Han W and Lo HW: Landscape of EGFR signaling network in human cancers: biology and therapeutic response in relation to receptor subcellular locations. Cancer Lett 318: 124-134, 2012.

40. Laubenbacher R, Hower V, Jarrah A, et al: A systems biology view of cancer. Biochim Biophys Acta 1796: 129-139, 2009.

41. Sharma SV and Settleman J: ErbBs in lung cancer. Exp Cell Res 315: 557-571, 2009.

42. Chiang YJ, Difilippantonio MJ, Tessarollo L, Morse HC and Hodes RJ: Exon 1 disruption alters tissue-specific expression of mouse p53 and results in selective development of B cell lymphomas. PLoS One 7: e49305, 2012. 
43. Erler JT and Linding R: Network medicine strikes a blow against breast cancer. Cell 149: 731-733, 2012.

44. Lee MJ, Ye AS, Gardino AK, et al: Sequential application of anticancer drugs enhances cell death by rewiring apoptotic signaling networks. Cell 149: 780-794, 2012.

45. Pilpel Y, Sudarsanam $P$ and Church GM: Identifying regulatory networks by combinatorial analysis of promoter elements. Nat Genet 29: 153-159, 2001.

46. Bhan A, Galas DJ and Dewey TG: A duplication growth model of gene expression networks. Bioinformatics 18: 1486-1493, 2002.

47. Pastor-Satorras R, Smith E and Sole RV: Evolving protein interaction networks through gene duplication. J Theor Biol 222: 199-210, 2003.

48. Gilmartin AG, Bleam MR, Groy A, et al: GSK1120212 (JTP-74057) is an inhibitor of MEK activity and activation with favorable pharmacokinetic properties for sustained in vivo pathway inhibition. Clin Cancer Res 17: 989-1000, 2011.

49. Duncan JS, Whittle MC, Nakamura K, et al: Dynamic reprogramming of the kinome in response to targeted MEK inhibition in triple-negative breast cancer. Cell 149: 307-321, 2012.

50. Slamon DJ, Clark GM, Wong SG, Levin WJ, Ullrich A and McGuire WL: Human breast cancer: correlation of relapse and survival with amplification of the HER-2/neu oncogene. Science 235: 177-182, 1987.

51. Hudis CA: Trastuzumab--mechanism of action and use in clinical practice. N Engl J Med 357: 39-51, 2007.

52. Crawford A and Nahta R: Targeting Bcl-2 in herceptin-resistant breast cancer cell lines. Curr Pharmacogenomics Person Med 9: 184-190, 2011.

53. Chan CH, Li CF, Yang WL, et al: The Skp2-SCF E3 ligase regulates Akt ubiquitination, glycolysis, herceptin sensitivity, and tumorigenesis. Cell 149: 1098-1111, 2012.

54. Figlin RA, Kaufmann I and Brechbiel J: Targeting PI3K and mTORC2 in metastatic renal cell carcinoma: new strategies for overcoming resistance to VEGFR and mTORC1 inhibitors. Int J Cancer 133: 788-796, 2013.

55. Li L, Story M and Legerski RJ: Cellular responses to ionizing radiation damage. Int J Radiat Oncol Biol Phys 49: 1157-1162, 2001.

56. Li YH, Wang X, Pan Y, Lee DH, Chowdhury D and Kimmelman AC: Inhibition of non-homologous end joining repair impairs pancreatic cancer growth and enhances radiation response. PLoS One 7: e39588, 2012.

57. Sonoda E, Hochegger H, Saberi A, Taniguchi Y and Takeda S: Differential usage of non-homologous end-joining and homologous recombination in double strand break repair. DNA Repair 5: 1021-1029, 2006.

58. Lhakhang TW and Chaudhry MA: Interactome of radiationinduced microRNA-predicted target genes. Comp Funct Genomics 2012: 569731, 2012.

59. Ma L, Nie L, Liu J, et al: An RNA-seq-based gene expression profiling of radiation-induced tumorigenic mammary epithelial cells. Genomics Proteomics Bioinformatics 10: 326-335, 2012.

60. Lee YS, Oh JH, Yoon S, et al: Differential gene expression profiles of radioresistant non-small-cell lung cancer cell lines established by fractionated irradiation: tumor protein p53-inducible protein 3 confers sensitivity to ionizing radiation. Int J Radiat Oncol Biol Phys 77: 858-866, 2010.

61. Kalanxhi E and Dahle J: Genome-wide microarray analysis of human fibroblasts in response to gamma radiation and the radiation-induced bystander effect. Radiat Res 177: 35-43, 2012.

62. Kim KH, Yoo HY, Joo KM, et al: Time-course analysis of DNA damage response-related genes after in vitro radiation in $\mathrm{H} 460$ and H1229 lung cancer cell lines. Exp Mol Med 43: 419-426, 2011.
63. Xu QY, Gao Y, Liu Y, Yang WZ and Xu XY: Identification of differential gene expression profiles of radioresistant lung cancer cell line established by fractionated ionizing radiation in vitro. Chin Med J 121: 1830-1837, 2008.

64. Ding LH, Shingyoji M, Chen F, et al: Gene expression profiles of normal human fibroblasts after exposure to ionizing radiation: a comparative study of low and high doses. Radiat Res 164: 17-26, 2005.

65. Rashi-Elkeles S, Elkon R, Shavit S, et al: Transcriptional modulation induced by ionizing radiation: p53 remains a central player. Mol Oncol 5: 336-348, 2011.

66. Tusher VG, Tibshirani R and Chu G: Significance analysis of microarrays applied to the ionizing radiation response. Proc Natl Acad Sci USA 98: 5116-5121, 2001.

67. Somosy Z: Radiation response of cell organelles. Micron 31: 165-181, 2000.

68. Cao N, Li S, Wang Z, et al: NF-kappaB-mediated HER2 overexpression in radiation-adaptive resistance. Radiat Res 171: 9-21, 2009.

69. Lee SY, Park HR, Cho NH, et al: Identifying genes related to radiation resistance in oral squamous cell carcinoma cell lines. Int J Oral Max Surg 42: 169-176, 2013.

70. Multhoff $\mathrm{G}$ and Radons J: Radiation, inflammation, and immune responses in cancer. Front Oncol 2: 58, 2012.

71. Brown JM and Wilson WR: Exploiting tumour hypoxia in cancer treatment. Nat Rev Cancer 4: 437-447, 2004.

72. Moeller BJ, Cao Y, Li CY and Dewhirst MW: Radiation activates HIF-1 to regulate vascular radiosensitivity in tumors: role of reoxygenation, free radicals, and stress granules. Cancer cell 5: 429-441, 2004.

73. Moeller BJ and Dewhirst MW: HIF-1 and tumour radiosensitivity. Br J Cancer 95: 1-5, 2006.

74. Kitano H: Biological robustness. Nature Rev Genet 5: 826-837, 2004.

75. Russell RB and Aloy P: Targeting and tinkering with interaction networks. Nat Chem Biol 4: 666-673, 2008

76. Shao L, Wang L, Wei Z, et al: Dynamic network of transcription and pathway crosstalk to reveal molecular mechanism of MGd-treated human lung cancer cells. PLoS One 7: e31984, 2012.

77. Komurov K, Tseng JT, Muller M, et al: The glucose-deprivation network counteracts lapatinib-induced toxicity in resistant ErbB2-positive breast cancer cells. Mol Syst Biol 8: 596, 2012.

78. Johnston S, Pippen J Jr, Pivot X, et al: Lapatinib combined with letrozole versus letrozole and placebo as first-line therapy for postmenopausal hormone receptor-positive metastatic breast cancer. J Clin Oncol 27: 5538-5546, 2009.

79. Eschrich S, Zhang H, Zhao H, et al: Systems biology modeling of the radiation sensitivity network: a biomarker discovery platform. Int J Radiat Oncol Biol Phys 75: 497-505, 2009.

80. Chiba M: Radiation-responsive transcriptome analysis in human lymphoid cells. Radiat Prot Dosimetry 152: 164-167, 2012.

81. Stiubea-Cohen R, David R, Neumann Y, et al: Effect of irradiation on cell transcriptome and proteome of rat submandibular salivary glands. PLoS One 7: e40636, 2012.

82. Russell JS, Brady K, Burgan WE, et al: Gleevec-mediated inhibition of Rad51 expression and enhancement of tumor cell radiosensitivity. Cancer Res 63: 7377-7383, 2003.

83. Raderschall E, Stout K, Freier S, Suckow V, Schweiger S and Haaf T: Elevated levels of Rad51 recombination protein in tumor cells. Cancer Res 62: 219-225, 2002.

84. Slupianek A, Hoser G, Majsterek I, et al: Fusion tyrosine kinases induce drug resistance by stimulation of homologydependent recombination repair, prolongation of $\mathrm{G}(2) / \mathrm{M}$ phase, and protection from apoptosis. Mol Cell Biol 22: 4189-4201, 2002 . 